\title{
The Geodetic Parameters of Strong Product Graphs
}

\author{
Ashalatha K.S \\ Research Scholar,Sri Gauthama Research Center, \\ (Affiliated to Kuvempu university),Government First Grade College, \\ Gubbi, Tumkur,Karnataka,India \\ Venkanagouda M Goudar \\ Sri Gauthama Research Center, Department of Mathematics, \\ Sri Siddhartha Institute of Technology, Tumkur, \\ Karnataka, India \\ Venkatesha \\ Department of Mathematics, Kuvempu University \\ Shankarghatta, Shimoga, Karnataka, India.
}

\begin{abstract}
A set $S \subseteq V(G)$ is a split geodetic set of $G$, if $S$ is a geodetic set and $\langle V-S\rangle$ is disconnected. The split geodetic number of a graph $G$, is denoted by $g_{s}(G)$, is the minimum cardinality of a split geodetic set of $G$. A set $S \subseteq V(G)$ is a strong split geodetic set of $G$, if $S$ is a geodetic set and $\langle V-S\rangle$ is totally disconnected. The strong split geodetic number of a graph $G$, is denoted by $g_{s s}(G)$, is the minimum cardinality of a strong split geodetic set of $G$. In this paper we obtain the geodetic number, split geodetic number, strong split geodetic number and non split geodetic number of strong product graphs, composition of graphs and join of graphs.
\end{abstract}

\section{Keywords:}

Cartesian product, Distance, Edge covering number, Split geodetic number, Vertex covering number

\section{INTRODUCTION}

In this paper we follow the notations of [4]. As usual $n=|V|$ and $m=|E|$ denote the number of vertices and edges of a graph $\mathrm{G}$ respectively.

The graphs considered here have at least one component which is not complete or at least two non trivial components.

The distance $d(u, v)$ between two vertices $\mathrm{u}$ and $\mathrm{v}$ in a connected graph $\mathrm{G}$ is the length of a shortest $u-v$ path in G. It is well known that this distance is a metric on the vertex set $V(G)$. For a vertex $v$ of $\mathrm{G}$, the eccentricity $e(v)$ is the distance between $v$ and a vertex farthest from $v$. The minimum eccentricity among the vertices of $\mathrm{G}$ is radius, $\operatorname{rad} \mathrm{G}$, and the maximum eccentricity is the diameter, diam G. A $u-v$ path of length $\mathrm{d}(u, v)$ is called a $u-v$ geodesic. We define $I[u, v]$ to the set (interval) of all vertices lying on some $u-v$ geodesic of $\mathrm{G}$ and for a nonempty subset $\mathrm{S}$ of $V(G), I[S]=$ $\bigcup_{u, v \in S} I[u, v]$.

A set $\mathrm{S}$ of vertices of $\mathrm{G}$ is called a geodetic set in $\mathrm{G}$ if $I[S]=V(G)$, and a geodetic set of minimum cardinality is a minimum geodetic set. The cardinality of a minimum geodetic set in $\mathrm{G}$ is called the geodetic number of $\mathrm{G}$, and we denote it by $g(G)$.
Split geodetic number of a graph was studied by in [5]. A geodetic set $S$ of a graph $G=(V, E)$ is a split geodetic set if the induced subgraph $\langle V-S\rangle$ is disconnected. The split geodetic number $g_{s}(G)$ of $G$ is the minimum cardinality of a split geodetic set. Strong split geodetic number of a graph was studied by in [1]. A set $S^{\prime}$ of vertices of $G=(V, E)$ is called the strong split geodetic set if the induced subgraph $\left\langle V-S^{\prime}\right\rangle$ is totally disconnected and a strong split geodetic set of minimum cardinality is the strong split geodetic number of $G$ and is denoted by $g_{s s}(G)$. Non split geodetic number of a graph was studied by in [6]. A geodetic set $S$ of a graph $G=(V, E)$ is a non split geodetic set if the induced subgraph $\langle V-S\rangle$ is connected. The non split geodetic number $g_{n s}(G)$ of $G$ is the minimum cardinality of a non split geodetic set.

The strong product of graphs $G_{1}$ and $G_{2}$, denoted by $G_{1} \otimes G_{2}$, has vertex set $V\left(G_{1}\right) \times V\left(G_{2}\right)$, where two distinct vertices $\left(x_{1}, y_{1}\right)$ and $\left(x_{2}, y_{2}\right)$ are adjacent with respect to the strong product if
(a) $x_{1}=x_{2}$ and $y_{1} y_{2} \in E\left(G_{2}\right)$ or
(b) $y_{1}=y_{2}$ and $x_{1} x_{2} \in E\left(G_{1}\right)$ or
(c) $x_{1} x_{2} \in E\left(G_{1}\right)$ and $y_{1} y_{2} \in E\left(G_{2}\right)$.
For any undefined term in this paper, see [3] and [4].

\section{PRELIMINARY NOTES}

We need the following results to prove further results.

THEOREM 2.1. [2] Every geodetic set of a graph contains its extreme vertices.

THEOREM 2.2. [5] For the wheel $W_{n}=K_{1}+C_{n-1}(n \geq$ $6)$,

$$
g_{s}\left(W_{n}\right)= \begin{cases}\frac{n+2}{2} & \text { if } n \text { is even } \\ \frac{n+1}{2} & \text { if } n \text { is odd }\end{cases}
$$


Volume 101 - No. 12, September 2014

THEOREM 2.3. [1] For the wheel $W_{n}=K_{1}+C_{n-1}(n \geq$ $6)$,

$$
g_{s s}\left(W_{n}\right)= \begin{cases}\frac{n+2}{2} & \text { if } n \text { is even } \\ \frac{n+1}{2} & \text { if } n \text { is odd } .\end{cases}
$$

Proposition 2.4. For any graph $G, g_{s}(G) \leq g_{s s}(G)$.

\section{MAIN RESULTS}

THEOREM 3.1. For any path $P_{n}$ of order $n>5, g\left(K_{2} \otimes\right.$ $\left.P_{n}\right)=4$.

Proof. Let $K_{2} \otimes P_{n}$ be formed from two copies of $G_{1}$ and $G_{2}$ of $P_{n}$. Let $U=\left\{u_{1}, u_{2}, \ldots, u_{n}\right\} \in V\left(G_{1}\right), W=\left\{w_{1}, w_{2}, \ldots, w_{n}\right\}$ $\in V\left(G_{2}\right)$ and $V=U \cup W$. Let $S=\left\{u_{1}, u_{n}, w_{1}, w_{n}\right\}$ be the geodetic set of $K_{2} \otimes P_{n}$, where $d\left(u_{1}, u_{n}\right)=\operatorname{diam}\left(K_{2} \otimes P_{n}\right)=$ $d\left(w_{1}, w_{n}\right)$, which covers all the vertices of $K_{2} \otimes P_{n}$. If possible let $P=\left\{u_{1}, u_{n}, w_{1}\right\},|P|<|S|$ be set of vertices, for any $w_{i} \in$ $V\left(K_{2} \otimes P_{n}\right), w_{i} \notin I[P]$ hence $P$ is not a geodetic set. Thus $S$ is the minimum geodetic set, there fore $g\left(K_{2} \otimes P_{n}\right)=4$.

THEOREM 3.2. For any path $P_{n}$ of order $n>5, g_{s}\left(K_{2} \otimes\right.$ $\left.P_{n}\right)=6$.

Proof. Let $K_{2} \otimes P_{n}$ be formed from two copies of $G_{1}$ and $G_{2}$ of $P_{n}$. Let $U=\left\{u_{1}, u_{2}, \ldots, u_{n}\right\} \in V\left(G_{1}\right), W=\left\{w_{1}, w_{2}, \ldots, w_{n}\right\}$ $\in V\left(G_{2}\right)$ and $V=U \cup W$. Let $S=H_{1} \cup H_{2}$, where $H_{1}=$ $\left\{u_{1}, u_{n}, w_{1}, w_{n}\right\} \subseteq V\left(K_{2} \otimes P_{n}\right)$ which covers all the vertices of $K_{2} \otimes P_{n}$ and $H_{2}=\left(u_{i}, w_{i}\right) \in E\left(K_{2} \otimes P_{n}\right) \subseteq V-H_{1}, u_{i}$ and $w_{i}$ are the vertices having maximum degree i.e $\operatorname{deg}\left(u_{i}\right)=\operatorname{deg}\left(w_{i}\right)=$ 5 . Now $S$ be the set of vertices which covers all the vertices of $K_{2} \otimes P_{n}$. Such that $V-S$ has more then one component. Then by the above argument $S$ is the minimal split geodetic set of $K_{2} \otimes P_{n}$. Clearly it follows that $|S|=\left|H_{1} \cup H_{2}\right|=4+2=6$. There fore $g_{s}\left(K_{2} \otimes P_{n}\right)=6$.

THEOREM 3.3. For any path $P_{n}$ of order $n \geq 5$

$$
g_{s s}\left(K_{2} \otimes P_{n}\right)\left\{\begin{array}{cc}
=4+n-2+\left\lfloor\frac{n}{3}\right\rfloor & n=5,6,7 \\
\geq 4+n-2+\left\lceil\frac{n}{3}\right\rceil & n \geq 8 .
\end{array}\right.
$$

Proof. Let $K_{2} \otimes P_{n}$ be formed from two copies of $G_{1}$ and $G_{2}$ of $P_{n}$. Let $U=\left\{u_{1}, u_{2}, \ldots, u_{n}\right\} \in V\left(G_{1}\right), W=\left\{w_{1}, w_{2}, \ldots, w_{n}\right\}$ $\in V\left(G_{2}\right)$ and $V=U \cup W$.

We have the following results.

Case i. Let $n=5,6,7$.

Consider $S=H_{1} \cup H_{2} \cup H_{3}$, where $H_{1}=\left\{u_{1}, u_{n}, w_{1}, w_{n}\right\} \subseteq$ $V\left(K_{2} \otimes P_{n}\right)$, which covers all the vertices of $K_{2} \otimes P_{n}, H_{2}=$ $\left\{w_{2}, w_{3}, \ldots, w_{n-1}\right\} \subseteq V-H_{1},\left|H_{2}\right|=n-2$ and $H_{3}=$ $\left\{w_{3}, w_{5}, \ldots, w_{i}\right\} \subseteq \bar{V}-H_{1},\left|H_{3}\right|=\left\lfloor\frac{n}{3}\right\rfloor$. Now $S$ be the set of vertices which covers all the vertices of $K_{2} \otimes P_{n}$, such that $V-S$ is totally disconnected. Then by the above argument $S$ is a minimal strong split geodetic set of $K_{2} \otimes P_{n}$. Clearly $|S|=\mid H_{1} \cup H_{2} \cup$ $H_{3} \mid=4+n-2+\left\lfloor\frac{n}{3}\right\rfloor$. There fore $g_{s s}\left(K_{2} \otimes P_{n}\right)=4+n-2+\left\lfloor\frac{n}{3}\right\rfloor$. Case ii. Let $n \geq 8$.

Consider $S=H_{1} \cup H_{2} \cup H_{3}$, where $H_{1}=\left\{u_{1}, u_{n}, w_{1}, w_{n}\right\} \subseteq$ $V\left(K_{2} \otimes P_{n}\right)$, which covers all the vertices of $K_{2} \otimes P_{n}, H_{2}=$ $\left\{w_{2}, w_{3}, \ldots, w_{n-1}\right\} \subseteq V-H_{1},\left|H_{2}\right|=n-2$ and $H_{3}=$ $\left\{w_{3}, w_{5}, \ldots, w_{i}\right\} \subseteq V-H_{1},\left|H_{3}\right| \geq\left\lceil\frac{n}{3}\right\rceil$. Now $S$ be the set of vertices which covers all the vertices of $K_{2} \otimes P_{n}$, such that $V-S$ is totally disconnected. Then by the above argument $S$ is a minimal strong split geodetic set of $K_{2} \otimes P_{n}$. Clearly $|S|=\mid H_{1} \cup H_{2} \cup$ $H_{3} \mid \geq 4+n-2+\left\lceil\frac{n}{3}\right\rceil$. There fore $g_{s s}\left(K_{2} \otimes P_{n}\right) \geq 4+n-2+\left\lceil\frac{n}{3}\right\rceil$.

THEOREM 3.4. For any path $P_{n}$ of order $n>5, g_{n s}\left(K_{2} \otimes\right.$ $\left.P_{n}\right)=4$.

Proof. Let $K_{2} \otimes P_{n}$ be formed from two copies of $G_{1}$ and $G_{2}$ of $P_{n}$. Let $U=\left\{u_{1}, u_{2}, \ldots, u_{n}\right\} \in V\left(G_{1}\right), W=\left\{w_{1}, w_{2}, \ldots, w_{n}\right\}$ $\in V\left(G_{2}\right)$ and $V=U \cup W$. Let $S=\left\{u_{1}, u_{n}, w_{1}, w_{n}\right\}$ be the non split geodetic set of $K_{2} \otimes P_{n}$, where $d\left(u_{1}, u_{n}\right)=\operatorname{diam}\left(K_{2} \otimes\right.$ $\left.P_{n}\right)=d\left(w_{1}, w_{n}\right)$, which covers all the vertices of $K_{2} \otimes P_{n}$ such that $V-S$ is connected. If possible let $P=\left\{u_{1}, u_{n}, w_{1}\right\},|P|<$ $|S|$ be set of vertices, for any $w_{i} \in V\left(K_{2} \otimes P_{n}\right), w_{i} \notin I[P]$ hence $P$ is not a geodetic set. Thus $S$ is the minimum non split geodetic set, there fore $g_{n s}\left(K_{2} \otimes P_{n}\right)=4$.

THEOREM 3.5. For any cycle $C_{n}$ of order $n>3$,

$$
g\left(K_{2} \otimes C_{n}\right)=\left\{\begin{array}{l}
4 \text { if } n \text { is even } \\
6 \text { if } n \text { is odd } .
\end{array}\right.
$$

Proof. Let $K_{2} \otimes C_{n}$ be formed from two copies of $G_{1}$ and $G_{2}$ of $C_{n}$. Let $U=\left\{u_{1}, u_{2}, \ldots, u_{n}\right\} \in V\left(G_{1}\right), W=\left\{w_{1}, w_{2}, \ldots, w_{n}\right\}$ $\in V\left(G_{2}\right)$ and $V=U \cup W$.

We have the following results.

Case i. Let $n$ be even.

Consider $S=\left\{u_{i}, u_{j}, w_{i}, w_{j}\right\}$ be the set of vertices such that $d\left(u_{i}, u_{j}\right)=\operatorname{diam}\left(K_{2} \otimes C_{n}\right)=d\left(w_{i}, w_{j}\right)$ and $\left\{\left(u_{i}, w_{i}\right),\left(u_{j}, w_{j}\right)\right\} \in E\left(K_{2} \otimes C_{n}\right)$, which covers all the vertices of $K_{2} \otimes C_{n}$. Let $P=\left\{u_{i}, u_{j}, w_{i}\right\},|P|<|S|$, for any $w_{k} \in V\left(K_{2} \otimes C_{n}\right), w_{k} \notin I[P]$. Hence $P$ is not a geodetic set. Thus $S$ is the minimum geodetic set of $K_{2} \otimes C_{n}$. There fore $g\left(K_{2} \otimes C_{n}\right)=4$.

Case ii. Let $n$ be odd.

Consider $S=\left\{u_{i}, u_{j}, u_{k}, w_{i}, w_{j}\right\}$ be the set of vertices such that $d\left(u_{i}, u_{j}\right)=d\left(u_{j}, u_{k}\right)=\operatorname{diam}\left(K_{2} \otimes C_{n}\right)=$ $d\left(w_{i}, w_{j}\right)=d\left(w_{j}, w_{k}\right)$ and $\left\{\left(u_{i}, w_{i}\right),\left(u_{j}, w_{j}\right),\left(u_{k}, w_{k}\right)\right\} \in$ $E\left(K_{2} \otimes C_{n}\right)$, which covers all the vertices of $K_{2} \otimes C_{n}$. Let $P=\left\{u_{i}, u_{j}, u_{k}, w_{i}, w_{j}\right\},|P|<|S|$, for any $w_{l} \in V\left(K_{2} \otimes C_{n}\right)$, $w_{l} \notin I[P]$. Hence $P$ is not a geodetic set. Thus $S$ is the minimum geodetic set of $K_{2} \otimes C_{n}$. There fore $g\left(K_{2} \otimes C_{n}\right)=6$.

THEOREM 3.6. For any cycle $C_{n}$ of order $n>3$,

$$
g_{s}\left(K_{2} \otimes C_{n}\right)=\left\{\begin{array}{l}
4 \text { if } n \text { is even } \\
6 \text { if } n \text { is odd. }
\end{array}\right.
$$

Proof. Let $K_{2} \otimes C_{n}$ be formed from two copies of $G_{1}$ and $G_{2}$ of $C_{n}$. Let $U=\left\{u_{1}, u_{2}, \ldots, u_{n}\right\} \in V\left(G_{1}\right), W=\left\{w_{1}, w_{2}, \ldots, w_{n}\right\}$ $\in V\left(G_{2}\right)$ and $V=U \cup W$.

We have the following results.

Case i. Let $n$ be even.

Consider $S=\left\{u_{i}, u_{j}, w_{i}, w_{j}\right\}$ be the split geodetic set, where $d\left(u_{i}, u_{j}\right)=\operatorname{diam}\left(K_{2} \otimes C_{n}\right)=d\left(w_{i}, w_{j}\right)$ and $\left\{\left(u_{i}, w_{i}\right),\left(u_{j}, w_{j}\right)\right\} \in E\left(K_{2} \otimes C_{n}\right)$, which covers all the vertices of $K_{2} \otimes C_{n}$, such that $V-S$ is disconnected. Let $P=\left\{u_{i}, u_{j}, w_{i}\right\}$, $|P|<|S|$, for any $w_{k} \in V\left(K_{2} \otimes C_{n}\right), w_{k} \notin I[P]$. Hence $P$ is not a geodetic set. Thus $S$ is the minimal split geodetic set of $K_{2} \otimes C_{n}$. There fore $g_{s}\left(K_{2} \otimes C_{n}\right)=4$.

Case ii. Let $n$ be odd. 
Volume 101 - No. 12, September 2014

Consider $S=\left\{u_{i}, u_{j}, u_{k}, w_{i}, w_{j}\right\}$ be the split geodetic set, where $d\left(u_{i}, u_{j}\right)=d\left(u_{j}, u_{k}\right)=\operatorname{diam}\left(K_{2} \otimes C_{n}\right)=d\left(w_{i}, w_{j}\right)=$ $d\left(w_{j}, w_{k}\right)$ and $\left\{\left(u_{i}, w_{i}\right),\left(u_{j}, w_{j}\right),\left(u_{k}, w_{k}\right)\right\} \in E\left(K_{2} \nabla C_{n}\right)$, which covers all the vertices of $K_{2} \otimes C_{n}$, such that $V-S$ is disconnected. Let $P=\left\{u_{i}, u_{j}, u_{k}, w_{i}, w_{j}\right\},|P|<|S|$, for any $w_{l} \in V\left(K_{2} \otimes C_{n}\right), w_{l} \notin I[P]$. Hence $P$ is not a geodetic set. Thus $S$ is the minimal split geodetic set of $K_{2} \otimes C_{n}$. There fore $g_{s}\left(K_{2} \otimes C_{n}\right)=6$.

THEOREM 3.7. For any cycle $C_{n}$ of order $n>3, g_{s s}\left(K_{2} \otimes\right.$ $\left.C_{n}\right)=2 n-4$.

Proof. Let $K_{2} \otimes C_{n}$ be formed from two copies of $G_{1}$ and $G_{2}$ of $C_{n}$. Let $U=\left\{u_{1}, u_{2}, \ldots, u_{n}\right\} \in V\left(G_{1}\right), W=\left\{w_{1}, w_{2}, \ldots, w_{n}\right\}$ $\in V\left(G_{2}\right)$ and $V=U \cup W$.

We have the following results.

Case i. Let $n$ be even.

Let $H_{1}=\left\{u_{i}, u_{j}, w_{i}, w_{j}\right\}$ be the minimum set of vertices which covers all the vertices of $K_{2} \otimes C_{n}$ by case i of Theorem 3.5. Now $S=H_{1} \cup H_{2}$, where $H_{2} \in V-H_{1}, V-H_{1}$ has two identical components and $\left|H_{2}\right|=2(n-4)$. Thus $I(S)=V\left(K_{2} \otimes C_{n}\right)$, clearly $V-S$ has independent set. Then by the above argument $S$ is a minimal strong split geodetic set of $K_{2} \otimes C_{n}$. Hence $|S|=$ $\left|H_{1} \cup H_{2}\right|=4+2(n-4)=2 n-4$. There fore $g_{s s}\left(K_{2} \otimes C_{n}\right)=$ $2 n-4$.

Case ii. Let $n$ be odd.

Let $H_{1}=\left\{u_{i}, u_{j}, u_{k}, w_{i}, w_{j}, w_{k}\right\}$ be the minimum set of vertices which covers all the vertices of $K_{2} \otimes C_{n}$ by case ii of Theorem 3.5. Now $S=H_{1} \cup H_{2}$, where $H_{2} \in V-H_{1}, V-H_{1}$ has two identical components and $\left|H_{2}\right|=2(n-5)$. Thus $I(S)=$ $V\left(K_{2} \otimes C_{n}\right)$, clearly $V-S$ has independent set. Then by the above argument $S$ is a minimal strong split geodetic set of $K_{2} \otimes C_{n}$. Hence $|S|=\left|H_{1} \cup H_{2}\right|=6+2(n-5)=2 n-4$. There fore $g_{s s}\left(K_{2} \otimes C_{n}\right)=2 n-4$.

THEOREM 3.8. $G^{\prime}$ be the graph obtained by adding an endedge $(x, y)$ to a cycle $C_{n}=G$ of order $n>3$, with $x \in G$ and $y \notin G$. Then

$$
g\left(K_{2} \otimes G^{\prime}\right)=\left\{\begin{array}{l}
4 \text { for even cycle } \\
6 \text { for odd cycle. }
\end{array}\right.
$$

Proof. Let $K_{2} \otimes G^{\prime}$ be formed from two copies of $G_{1}^{\prime}$ and $G_{2}^{\prime}$ of $G^{\prime}$. Let $U=\left\{u_{1}, u_{2}, \ldots, u_{n}\right\} \in V\left(G_{1}^{\prime}\right), W=\left\{w_{1}, w_{2}, \ldots, w_{n}\right\}$ $\in V\left(G_{2}^{\prime}\right)$ and $V=U \cup W$.

We have the following results.

Case i. For even cycle.

Let $S=\left\{u_{i}, u_{j}, w_{i}, w_{j}\right\}$ be the set of vertices such that $u_{i}$ and $w_{i}$ are the vertices formed from end-vertex of $G^{\prime},\left(u_{i}, w_{i}\right) \in$ $E\left(K_{2} \otimes G^{\prime}\right)$ and $u_{j}, w_{j}$ are the vertices formed from the antipodal vertex of $G$ corresponding to the vertex adjacent to end vertex, $\left(u_{j}, w_{j}\right) \in E\left(K_{2} \otimes G^{\prime}\right)$. Clearly $I(S)=V\left(K_{2} \otimes G^{\prime}\right)$. Thus $S$ is the minimal geodetic set. There fore $g\left(K_{2} \otimes G^{\prime}\right)=4$.

Case ii. For odd cycle.

Let $S=\left\{u_{i}, u_{j}, u_{k}, w_{i}, w_{j}, w_{k}\right\}$ be the set of vertices such that $u_{i}$ and $w_{i}$ are the vertices formed from end-vertex of $G^{\prime},\left(u_{i}, w_{i}\right) \in$ $E\left(K_{2} \otimes G^{\prime}\right)$ and $d\left(u_{i}, u_{j}\right)=2, d\left(u_{j}, u_{k}\right)=\left\lfloor\frac{n}{2}\right\rfloor$ similarly $d\left(w_{i}, w_{j}\right)=2, d\left(w_{j}, w_{k}\right)=\left\lfloor\frac{n}{2}\right\rfloor$ and $\left\{\left(u_{j}, w_{j}\right),\left(u_{k}, w_{k}\right)\right\} \in$ $E\left(K_{2} \otimes G^{\prime}\right)$. Clearly $I(S)=V\left(K_{2} \otimes G^{\prime}\right)$. Thus $S$ is the minimal geodetic set. There fore $g\left(K_{2} \otimes G^{\prime}\right)=6$.

\section{THEOREM 3.9. $G^{\prime}$ be the graph obtained by adding an end-} edge $(x, y)$ to a cycle $C_{n}=G$ of order $n>3$, with $x \in G$ and $y \notin G$. Then

$$
g_{s}\left(K_{2} \otimes G^{\prime}\right)=\left\{\begin{array}{l}
7 \text { for even cycle } \\
6 \text { for odd cycle. }
\end{array}\right.
$$

Proof. Let $K_{2} \otimes G^{\prime}$ be formed from two copies of $G_{1}^{\prime}$ and $G_{2}^{\prime}$ of $G^{\prime}$. Let $U=\left\{u_{1}, u_{2}, \ldots, u_{n}\right\} \in V\left(G_{1}^{\prime}\right), W=\left\{w_{1}, w_{2}, \ldots, w_{n}\right\}$ $\in V\left(G_{2}^{\prime}\right)$ and $V=U \cup W$.

We have the following results.

Case i. For even cycle.

Let $H_{1}=\left\{u_{i}, u_{j}, w_{i}, w_{j}\right\}$ be the minimum set of vertices which covers all the vertices of $K_{2} \otimes G^{\prime}$ by case i of Theorem 3.8. Consider $S=H_{1} \cup H_{2}$, where $H_{2}=\left\{u_{k}, w_{k}, w_{l}\right\} \subseteq V-H_{1}$ such that $V-S$ has more then one component. Then by the above argument $S$ is minimal split geodetic set of $K_{2} \otimes G^{\prime}$. Hence $|S|=\left|H_{1} \cup H_{2}\right|=4+3=7$. There fore $g_{s}\left(K_{2} \otimes G^{\prime}\right)=7$.

Case ii. For odd cycle.

Let $S=\left\{u_{i}, u_{j}, u_{k}, w_{i}, w_{j}, w_{k}\right\}$ be the minimal geodetic set by case ii of Theorem 3.8. Since $V-S$ has two components $S$ it self is the minimal split geodetic set of $K_{2} \otimes G^{\prime}$.There fore $g_{s}\left(K_{2} \otimes G^{\prime}\right)=6$.

THEOREM 3.10. $G^{\prime}$ be the graph obtained by adding an end-edge $(x, y)$ to a cycle $C_{n}=G$ of order $n>3$, with $x \in G$ and $y \notin G$. Then

$$
g_{s s}\left(K_{2} \otimes G^{\prime}\right)=\left\{\begin{array}{lr}
\frac{3 n+4}{2} & \text { for even cycle } \\
6+n-2+\left\lfloor\frac{n}{3}\right\rfloor & \text { for } n=5 \\
6+n & \text { for } n=7 \\
6+n-2+\left\lceil\frac{n}{3}\right\rceil & \text { for odd cycle. }
\end{array}\right.
$$

Proof. Let $K_{2} \otimes G^{\prime}$ be formed from two copies of $G_{1}^{\prime}$ and $G_{2}^{\prime}$ of $G^{\prime}$. Let $U=\left\{u_{1}, u_{2}, \ldots, u_{n}\right\} \in V\left(G_{1}^{\prime}\right), W=\left\{w_{1}, w_{2}, \ldots, w_{n}\right\}$ $\in V\left(G_{2}^{\prime}\right)$ and $V=U \cup W$.

We have the following results.

Case i. For even cycle.

Consider $S=H_{1} \cup H_{2}$ be the minimal strong split geodetic set of $K_{2} \otimes G^{\prime}$, where $H_{1}=\left\{u_{i}, u_{j}, w_{i}, w_{j}, u_{k}, w_{k}, w_{l}\right\}$, $I\left(H_{1}\right)=V\left(K_{2} \otimes G^{\prime}\right)$ by case $i$ of Theorem 3.9 and $H_{2} \subseteq V-H_{1}$, $\left|H_{2}\right|=\frac{3 n-10}{2}$, such that $V-S$ is totally disconnected. Hence $|S|=\left|H_{1} \cup H_{2}\right|=7+\frac{3 n-10}{2}=\frac{3 n+4}{2}$. There fore $g_{s s}\left(K_{2} \otimes G^{\prime}\right)=$ $\frac{3 n+4}{2}$.

Case ii. For $n=5$.

Consider $S=H_{1} \cup H_{2}$ be the minimal strong split geodetic set of $K_{2} \otimes G^{\prime}$, where $H_{1}=\left\{u_{i}, u_{j}, u_{k}, w_{i}, w_{j}, w_{k}\right\}$ be the set of vertices which covers all the vertices of $K_{2} \otimes G^{\prime}$ by case ii of Theorem 3.8 and $H_{2} \subseteq V-H_{1},\left|H_{2}\right|=n-2+\left\lfloor\frac{n}{3}\right\rfloor$, such that $V-S$ is totally disconnected. Hence $|S|=\left|H_{1} \cup H_{2}\right|=6+n-2+\left\lfloor\frac{n}{3}\right\rfloor$. There fore $g_{s s}\left(K_{2} \otimes G^{\prime}\right)=6+n-2+\left\lfloor\frac{n}{3}\right\rfloor$.

Case iii. For $n=7$.

Consider $S=H_{1} \cup H_{2}$ be the minimal strong split geodetic set of $K_{2} \otimes G^{\prime}$, where $H_{1}=\left\{u_{i}, u_{j}, u_{k}, w_{i}, w_{j}, w_{k}\right\}$ be the set of vertices which covers all the vertices of $K_{2} \otimes G^{\prime}$ by case ii of Theorem 3.8 and $H_{2} \subseteq V-H_{1},\left|H_{2}\right|=n$, such that $V-S$ is to- 
Volume 101 - No. 12, September 2014

tally disconnected. Hence $|S|=\left|H_{1} \cup H_{2}\right|=6+n$. There fore $g_{s s}\left(K_{2} \otimes G^{\prime}\right)=6+n$.

Case iv. For odd cycle $(n>7)$.

Consider $S=H_{1} \cup H_{2}$ be the minimal strong split geodetic set of $K_{2} \otimes G^{\prime}$, where $H_{1}=\left\{u_{i}, u_{j}, u_{k}, w_{i}, w_{j}, w_{k}\right\}$ be the set of vertices which covers all the vertices of $K_{2} \otimes G^{\prime}$ by case ii of Theorem 3.8 and $H_{2} \subseteq V-H_{1},\left|H_{2}\right|=n-2+\left\lceil\frac{n}{3}\right\rceil$, such that $V-S$ is totally disconnected. Hence $|S|=\left|H_{1} \cup H_{2}\right|=6+n-2+\left\lceil\frac{n}{3}\right\rceil$. There fore $g_{s s}\left(K_{2} \otimes G^{\prime}\right)=6+n-2+\left\lceil\frac{n}{3}\right\rceil$.

THEOREM 3.11. For the wheel $W_{n}=K_{1}+C_{n-1}(n \geq 6)$,

$$
g\left(K_{2} \otimes W_{n}\right)= \begin{cases}n & \text { if } n \text { is even } \\ n-1 & \text { if } n \text { is odd } .\end{cases}
$$

Proof. Let $K_{2} \otimes W_{n}$ be formed from two copies of $G_{1}$ and $G_{2}$ of $W_{n}$. Let $U=\left\{u_{1}, u_{2}, \ldots, u_{n}\right\} \in V\left(G_{1}\right), W=\left\{w_{1}, w_{2}, \ldots, w_{n}\right\}$ $\in V\left(G_{2}\right)$ and $V=U \cup W$.

We have the following results.

Case i. Let $n$ be even.

Consider the geodesic $P:\left\{u_{1}, u_{2}, u_{6}, w_{2}, w_{6}, u_{3}\right\}$, $Q \quad: \quad\left\{u_{3}, u_{4}, u_{6}, w_{4}, w_{6}, u 5\right\}, \ldots, \quad R \quad$ : $\left\{u_{n-3}, u_{n-2}, u_{n}, w_{n-2}, w_{n}, u_{n-1}\right\}$ and the geodesics, $H=\left\{w_{1}-w_{3}, w_{3}-w_{5}, \ldots, w_{n-3}-w_{n-1}\right\}$ It is clear that the vertices $u_{2}, u_{4}, u_{6}, \ldots, u_{n-2}, w_{2}, w_{4}, w_{6}, \ldots, w_{n-2}$ lies on the geodesics $P, Q, R$ and $H$. Thus the set $S=\left\{u_{1}, u_{3}, \ldots, u_{n-1}, w_{1}, w_{3}, \ldots, w_{n-1}\right\}$ is the minimum geodetic set $K_{2} \otimes W_{n}$. There fore $g\left(K_{2} \otimes W_{n}\right)=|S|=n$.

Case ii. Let $n$ be odd.

Consider the geodesic $P:\left\{u_{1}, u_{2}, u_{7}, w_{2}, w_{7}, u_{3}\right\}$, $Q \quad: \quad\left\{u_{3}, u_{4}, u_{7}, w_{4}, w_{7}, u 5\right\}, \ldots, \quad R$ : $\left\{u_{n-4}, u_{n-3}, u_{n}, w_{n-3}, w_{n}, u_{n-2}\right\} \quad$ and the geodesics, $H=\left\{w_{1}-w_{3}, w_{3}-w_{5}, \ldots, w_{n-4}-w_{n-2}\right\}$ It is clear that the vertices $u_{2}, u_{4}, u_{6}, \ldots, u_{n-1}, w_{2}, w_{4}, w_{6}, \ldots, w_{n-1}$ lies on the geodesics $P, Q, R$ and $H$. Thus the set $S=\left\{u_{1}, u_{3}, \ldots, u_{n-2}, w_{1}, w_{3}, \ldots, w_{n-2}\right\}$ is the minimum geodetic set $K_{2} \otimes W_{n}$. There fore $g\left(K_{2} \otimes W_{n}\right)=|S|=n-1$.

THEOREM 3.12. For the wheel $W_{n}=K_{1}+C_{n-1}(n \geq 6)$,

$$
g_{s}\left(K_{2} \otimes W_{n}\right)=\left\{\begin{array}{l}
n+2 \text { if } n \text { is even } \\
n+1 \text { if } n \text { is odd. }
\end{array}\right.
$$

Proof. Let $K_{2} \otimes W_{n}$ be formed from two copies of $G_{1}$ and $G_{2}$ of $W_{n}$. Let $U=\left\{u_{1}, u_{2}, \ldots, u_{n}\right\} \in V\left(G_{1}\right), W=\left\{w_{1}, w_{2}, \ldots, w_{n}\right\}$ $\in V\left(G_{2}\right)$ and $V=U \cup W$.

We have the following results.

Case i. Let $n$ be even.

Consider $S=H_{1} \cup H_{2} \quad$ where $H_{1}=$ $\left\{u_{1}, u_{3}, \ldots, u_{n-1}, w_{1}, w_{3}, \ldots, w_{n-1}\right\}$ be the minimum geodetic set by case $\mathrm{i}$ of Theorem 3.11 and $H_{2}=\left\{v_{i}, w_{i}\right\} \subseteq V\left(K_{2} \otimes W_{n}\right)$, formed from the vertex $K_{1}$ of $W_{n}$. Now $S$ is the minimal split geodetic set of $K_{2} \otimes W_{n}$, since $V-S$ has $\frac{n-2}{2}$ times $K_{2}$ components. Hence $|S|=\left|H_{1} \cup H_{2}\right|=n+2$. There fore $g_{s}\left(K_{2} \otimes W_{n}\right)=n+2$.

Case ii. Let $n$ be odd.

Consider $S=H_{1} \cup H_{2}$ where $H_{1}=$ $\left\{u_{1}, u_{3}, \ldots, u_{n-2}, w_{1}, w_{3}, \ldots, w_{n-2}\right\}$ be the minimum geodetic set by case ii of Theorem 3.11 and $H_{2}=\left\{v_{i}, w_{i}\right\} \subseteq V\left(K_{2} \otimes W_{n}\right)$, formed from the vertex $K_{1}$ of $W_{n}$. Now $S$ is the minimal split geodetic set of $K_{2} \otimes W_{n}$, since $V-S$ has $\frac{n-1}{2}$ times $K_{2}$ components. Hence $|S|=\left|H_{1} \cup H_{2}\right|=n-1+2=n+1$. There fore $g_{s}\left(K_{2} \otimes W_{n}\right)=n+1$.

THEOREM 3.13. For the wheel $W_{n}=K_{1}+C_{n-1}(n \geq 6)$,

$$
g_{s s}\left(K_{2} \otimes W_{n}\right)= \begin{cases}\frac{3 n+2}{2} & \text { if } n \text { is even } \\ \frac{3 n+1}{2} & \text { if } n \text { is odd. }\end{cases}
$$

Proof. Let $K_{2} \otimes W_{n}$ be formed from two copies of $G_{1}$ and $G_{2}$ of $W_{n}$. Let $U=\left\{u_{1}, u_{2}, \ldots, u_{n}\right\} \in V\left(G_{1}\right), W=\left\{w_{1}, w_{2}, \ldots, w_{n}\right\}$ $\in V\left(G_{2}\right)$ and $V=U \cup W$.

We have the following results.

Case i. Let $n$ be even.

Consider $H=H_{1} \cup H_{2}$ where $H_{1}=$ $\left\{u_{1}, u_{3}, \ldots, u_{n-1}, w_{1}, w_{3}, \ldots, w_{n-1}\right\}$ be the minimum geodetic set by case $\mathrm{i}$ of Theorem 3.11 and $H_{2}=\left\{v_{i}, w_{i}\right\} \subseteq V\left(K_{2} \otimes W_{n}\right)$, formed from the vertex $K_{1}$ of $W_{n}$, such that $V-H$ has $\frac{n-2}{2}$ times $K_{2}$ components. Let $S=H \cup H_{3}$, where $H_{3} \subseteq V-H$ consists of one vertex from each $K_{2}$ components, $\left|H_{3}\right|=\frac{n-2}{2}$. Now $S$ is the minimal strong split geodetic set of $K_{2} \otimes W_{n}^{2}$ since $V-S$ has isolated vertices. Hence $|S|=\left|H \cup H_{3}\right|=\left|H_{1} \cup H_{2} \cup H_{3}\right|=n+2+\frac{n-2}{2}=\frac{3 n+2}{2}$. There fore $g_{s s}\left(K_{2} \otimes W_{n}\right)=\frac{3 n+2}{2}$.

Case ii. Let $n$ be odd.

Consider $H=H_{1} \cup H_{2}$ where $H_{1}=$ $\left\{u_{1}, u_{3}, \ldots, u_{n-2}, w_{1}, w_{3}, \ldots, w_{n-2}\right\}$ be the minimum geodetic set by case ii of Theorem 3.11 and $H_{2}=\left\{v_{i}, w_{i}\right\} \subseteq V\left(K_{2} \otimes W_{n}\right)$, formed from the vertex $K_{1}$ of $W_{n}$, such that $V-H$ has $\frac{n-1}{2}$ times $K_{2}$ components. Let $S=H \cup H_{3}$, where $H_{3} \subseteq V-H^{2}$ consists of one vertex from each $K_{2}$ components, $\left|H_{3}\right|=\frac{n-1}{2}$. Now $S$ is the minimal strong split geodetic set of $K_{2} \otimes W_{n}{ }^{2}$ since $V-S$ has isolated vertices. Hence $|S|=\left|H \cup H_{3}\right|=\left|H_{1} \cup H_{2} \cup H_{3}\right|$ $=n-1+2+\frac{n-1}{2}=\frac{3 n+1}{2}$. There fore $g_{s s}\left(K_{2} \otimes W_{n}\right)=\frac{3 n+1}{2}$.

THEOREM 3.14. For the wheel $W_{n}=K_{1}+C_{n-1}(n \geq 6)$,

$$
g_{n s}\left(K_{2} \otimes W_{n}\right)= \begin{cases}n & \text { if } n \text { is even } \\ n-1 & \text { if } n \text { is odd. }\end{cases}
$$

Proof. Let $K_{2} \otimes W_{n}$ be formed from two copies of $G_{1}$ and $G_{2}$ of $W_{n}$. Let $U=\left\{u_{1}, u_{2}, \ldots, u_{n}\right\} \in V\left(G_{1}\right), W=\left\{w_{1}, w_{2}, \ldots, w_{n}\right\}$ $\in V\left(G_{2}\right)$ and $V=U \cup W$.

We have the following results.

Case i. Let $n$ be even.

Consider the geodesic $P:\left\{u_{1}, u_{2}, u_{6}, w_{2}, w_{6}, u_{3}\right\}$, $Q \quad: \quad\left\{u_{3}, u_{4}, u_{6}, w_{4}, w_{6}, u 5\right\} \quad, \ldots, \quad R \quad$ : $\left\{u_{n-3}, u_{n-2}, u_{n}, w_{n-2}, w_{n}, u_{n-1}\right\}$ and the geodesics, $H=\left\{w_{1}-w_{3}, w_{3}-w_{5}, \ldots, w_{n-3}-w_{n-1}\right\}$ It is clear that the vertices $u_{2}, u_{4}, u_{6}, \ldots, u_{n-2}, w_{2}, w_{4}, w_{6}, \ldots, w_{n-2}$ lies on the geodesics $P, Q, R$ and $H$. Thus the set $S=\left\{u_{1}, u_{3}, \ldots, u_{n-1}, w_{1}, w_{3}, \ldots, w_{n-1}\right\}$ is the minimum non split geodetic set $K_{2} \otimes W_{n}$, since $V-S$ is connected. There fore $g_{n s}\left(K_{2} \otimes W_{n}\right)=|S|=n$.

Case ii. Let $n$ be odd.

Consider the geodesic $P:\left\{u_{1}, u_{2}, u_{7}, w_{2}, w_{7}, u_{3}\right\}$, $Q \quad: \quad\left\{u_{3}, u_{4}, u_{7}, w_{4}, w_{7}, u 5\right\} \quad, \ldots, \quad R \quad$ : $\left\{u_{n-4}, u_{n-3}, u_{n}, w_{n-3}, w_{n}, u_{n-2}\right\} \quad$ and the geodesics, $H=\left\{w_{1}-w_{3}, w_{3}-w_{5}, \ldots, w_{n-4}-w_{n-2}\right\}$ It is clear that the vertices $u_{2}, u_{4}, u_{6}, \ldots, u_{n-1}, w_{2}, w_{4}, w_{6}, \ldots, w_{n-1}$ lies on the geodesics $P, Q, R$ and $H$. Thus the set $S=\left\{u_{1}, u_{3}, \ldots, u_{n-2}, w_{1}, w_{3}, \ldots, w_{n-2}\right\}$ is the minimum 
Volume 101 - No. 12, September 2014

THEOREM 4.1. For any cycle $C_{n}$ of order $n>3$,

$$
g\left(K_{2}\left[C_{n}\right]\right)=\left\{\begin{array}{l}
2 \quad \text { for } n=4 \\
3 \text { for } n=5,6 \\
4 \quad \text { for } n_{6} 6 .
\end{array}\right.
$$

Proof. Let $K_{2}\left[C_{n}\right]$ be formed from two copies of $G_{1}$ and $G_{2}$ of $C_{n}$. Let $U=\left\{u_{1}, u_{2}, \ldots, u_{n}\right\} \in V\left(G_{1}\right), W=\left\{w_{1}, w_{2}, \ldots, w_{n}\right\}$ $\in V\left(G_{2}\right)$ and $V=U \cup W$.

We have the following results.

Case i. Let $n=4$.

Consider $S=\left\{v_{1}, v_{3}\right\}$ be the set which covers all the vertices of $K_{2}\left[C_{n}\right]$, where $\left\{v_{1}, v_{3}\right\} \notin E\left(G_{1}\right)$. Which forms a minimum geodetic set of $K_{2}\left[C_{n}\right]$, therefore $g\left(K_{2}\left[C_{n}\right]\right)=2$.

Case ii. Let $n=5,6$.

Consider $S=\left\{v_{1}, v_{3}, v_{5}\right\}$ be the set which covers all the vertices of $K_{2}\left[C_{n}\right]$, where $\left\{\left(v_{1}, v_{3}\right),\left(v_{3}, v_{5}\right)\right\} \notin E\left(G_{1}\right)$ and $d\left(v_{1}, v_{3}\right)=$ $d\left(v_{3}, v_{5}\right)=\operatorname{diam}\left(K_{2}\left[C_{n}\right]\right)$. If possible let $P=\left\{v_{1}, v_{3}\right\} \in$ $V\left(K_{2}\left[C_{n}\right]\right),|P|<|S|$ be a set, for any $v_{i} \notin I[P]$. Thus $S$ ia minimal geodetic set $K_{2}\left[C_{n}\right]$. Therefore $g\left(K_{2}\left[C_{n}\right]\right)=3$.

Case iii. Let $n>6$.

Consider $S=\left\{v_{i}, v_{j}, w_{i}, w_{j}\right\}$ be the minimal geodetic set of $K_{2}\left[C_{n}\right]$, where $\left\{\left(v_{i}, v_{j}\right),\left(w_{i}, w_{j}\right)\right\} \notin E\left(K_{2}\left[C_{n}\right]\right)$ and $d\left(v_{i}, v_{j}\right)=d\left(w_{i}, w_{j}\right)=\operatorname{diam}\left(K_{2}\left[C_{n}\right]\right)$, which covers all the vertices of $K_{2}\left[C_{n}\right]$. Thus $g\left(K_{2}\left[C_{n}\right]\right)=4$.

THEOREM 4.2. For any cycle $C_{n}$ of order $n>3$,

$$
g_{s}\left(K_{2}\left[C_{n}\right]\right)=\left\{\begin{array}{lr}
\frac{2 n+4}{2} & \text { for } n=4,5,6 \\
n+2 & \text { for } n_{\zeta} 6
\end{array}\right.
$$

THEOREM 4.3. For any cycle $C_{n}$ of order $n>3$

$$
g_{s s}\left(K_{2}\left[C_{n}\right]\right)= \begin{cases}\frac{3 n}{2} & \text { if } n \text { is even } \\ \frac{3 n+1}{2} & \text { if } n \text { is odd } .\end{cases}
$$

Proof. Let $K_{2} \otimes T$ be formed from two copies of $G_{1}$ and $G_{2}$ of $T$. Let $U=\left\{u_{1}, u_{2}, \ldots, u_{n}\right\} \in V\left(G_{1}\right), W=\left\{w_{1}, w_{2}, \ldots, w_{n}\right\}$ $\in V\left(G_{2}\right)$ and $V=U \cup W$.

We have the following results.

Case i. Let $k$ be even.

Consider $S=H_{1} \cup H_{2}$, be the strong split geodetic set of $K_{2} \otimes$ $T$, where $H_{1}=\left\{u_{1}, u_{2}, \ldots, u_{k}, w_{1}, w_{2}, \ldots, w_{k}\right\} \subseteq V\left(K_{2} \otimes T\right)$ formed from the set of end-edges of $T, I\left(H_{1}\right)=V\left(K_{2} \otimes T\right)$ and $H_{2}=\left\{u_{i}, w_{i}, u_{j}, w_{j}, \ldots\right\} \subseteq V-H_{1},\left|H_{2}\right|=\frac{3 k}{2}$. Thus by the above argument $S$ is the minimal strong split geodetic set of $K_{2} \otimes T$. There fore $g_{s s}\left(K_{2} \otimes T\right)=|S|=\left|H_{1} \cup H_{2}\right|=2 k+\frac{3 k}{2}=$ $\frac{7 k}{2}$.

Case ii. Let $k$ be odd.

Consider $S=H_{1} \cup H_{2}$, be the strong split geodetic set of $K_{2} \otimes$ $T$, where $H_{1}=\left\{u_{1}, u_{2}, \ldots, u_{k}, w_{1}, w_{2}, \ldots, w_{k}\right\} \subseteq V\left(K_{2} \otimes T\right)$ formed from the set of end-edges of $T, I\left(H_{1}\right)=V\left(K_{2} \otimes T\right)$ and $H_{2}=\left\{u_{i}, w_{i}, u_{j}, w_{j}, \ldots\right\} \subseteq V-H_{1},\left|H_{2}\right|=\frac{3 k-1}{2}$. Thus by the above argument $S$ is the minimal strong split geodetic set of $K_{2} \otimes T$. There fore $g_{s s}\left(K_{2} \otimes T\right)=|S|=\left|H_{1} \cup H_{2}\right|=$ $2 k+\frac{3 k-1}{2}=\frac{7 k-1}{2}$.

\section{COMPOSITION OF GRAPHS}

The composition $G=G_{1}\left[G_{2}\right]$ has $V=V_{1} \times V_{2}$ as its point set, and $u=\left(u_{1}, u_{2}\right)$ is adjacent with $v=\left(v_{1}, v_{2}\right)$ whenever [ $u_{1}$ adj $\left.v_{1}\right]$ or $\left[u_{1}=v_{1}\right.$ and $u_{2}$ adj $\left.v_{2}\right]$.
THEOREM 4.4. $G$ ' be the graph obtained by adding an endedge $(x, y)$ to a cycle $C_{n}=G$ of order $n>3$, with $x \in G$ and $y \notin G$. Then $g\left(K_{2}\left[G^{\prime}\right]\right)=4$.

THEOREM 4.5. $G$ ' be the graph obtained by adding an endedge $(x, y)$ to a cycle $C_{n}=G$ of order $n>3$, with $x \in G$ and $y \notin G$. Then $g_{s}\left(K_{2}\left[G^{\prime}\right]\right)=n+3$

THEOREM 4.6. $G^{\prime}$ be the graph obtained by adding an endedge $(x, y)$ to a cycle $C_{n}=G$ of order $n>3$, with $x \in G$ and $y \notin G$. Then

$$
g_{s s}\left(K_{2}\left[G^{\prime}\right]\right)=\left\{\begin{array}{l}
\frac{3 n+2}{2} \text { for even cycle } \\
\frac{3 n+3}{2} \text { for odd cycle. }
\end{array}\right.
$$

\section{JOIN OF GRAPHS}

The join of two graphs $G_{1}$ and $G_{2}$, written as $G_{1}+G_{2}$, is defined as the union of $G_{1}$ and $G_{2}$ together with all edges $(u, v)$ for which $u \in V\left(G_{1}\right)$ and $v \in V\left(G_{2}\right)$. Two vertices of a graph $G$ are said to be joined in $G$ if the edge $(u, v)$ is contained in the edge set of $G$. 
THEOREM 5.1. For any cycle $C_{n}$ of order $n>3$,

$$
g\left(K_{2}+C_{n}\right)= \begin{cases}\frac{n}{2} & \text { if } n \text { is even } \\ \frac{n+1}{2} & \text { if } n \text { is odd } .\end{cases}
$$

THEOREM 5.2. For any cycle $C_{n}$ of order $n>3$,

$$
g_{s}\left(K_{2}+C_{n}\right)=g_{s s}\left(K_{2}+C_{n}\right)= \begin{cases}\frac{n+4}{2} & \text { if } n \text { is even } \\ \frac{n+5}{2} & \text { if } n \text { is odd } .\end{cases}
$$

THEOREM 5.3. $G^{\prime}$ be the graph obtained by adding an endedge $(x, y)$ to a cycle $C_{n}=G$ of order $n>3$, with $x \in G$ and $y \notin G$. Then

$$
g\left(K_{2}+G^{\prime}\right)= \begin{cases}\frac{n+2}{2} & \text { for even cycle } \\ \frac{n+3}{2} & \text { for odd cycle. }\end{cases}
$$

THEOREM 5.4. $G^{\prime}$ be the graph obtained by adding an endedge $(x, y)$ to a cycle $C_{n}=G$ of order $n>3$, with $x \in G$ and $y \notin G$. Then

$$
g_{s}\left(K_{2}+G^{\prime}\right)=g_{s s}\left(K_{2}+G^{\prime}\right)=\left\{\begin{array}{l}
\frac{n+6}{2} \text { for even cycle } \\
\frac{n+7}{2} \text { for odd cycle. }
\end{array}\right.
$$

\section{CONCLUSION}

In this paper we have establish many results on split geodetic number, nonsplit geodetic number, strong split geodetic number of strong product of graph and some observation on split geodetic number, nonsplit geodetic number, strong split geodetic number of composition graphs and join of graphs.

\section{ACKNOWLEDGEMENT}

Our thanks to the referee's for the help in revising the manuscript.

\section{REFERENCES}

[1] Ashalatha K.S., Venkanagouda. M. Goudar, Venkatesha., 2014. Strong split geodetic number of a graph, International Journal of Computer Applications., 89 (4) (2014), 1-4

[2] G. Chartrand, F. Harary, and P.Zhang, 2002.

On the geodetic number of a graph.Networks.39, (2002), 1-6.

[3] G. Chartrand and P.Zhang, 2006.

Introduction to Graph Theory, Tata McGraw Hill Pub.Co.Ltd.

[4] F.Harary, 1969. Graph Theory, Addison-Wesely, Reading, MA.

[5] Venkanagouda M.Goudar, K.S.Ashalatha, Venkatesha, 2014. Split Geodetic Number of a Graph, Advances and Applications in Discrete Mathematics. 13(1) (2014), 9-22.
[6] Venkanagouda. M. Goudar, Tejaswini K. M, Venkatesha., Non split geodetic number of a graph, Indian Journal of Pure and Applied

Mathematics. (Communicated). 